\section{GENRE} en séries

\section{Genre en séries}

Cinéma, télévision, médias

$6 \mid 2017$

Être mère, être père : représentations et discours médiatiques

\title{
Visibiliser et normaliser les familles homoparentales en Espagne. Les actions médiatiques de l'association Familles Lesbiennes et gays
}

\author{
Marta Roca i Escoda
}

\section{OpenEdition \\ Journals}

\section{Édition électronique}

URL : http://journals.openedition.org/ges/953

DOI : $10.4000 /$ ges.953

ISSN : 2437-6563

\section{Éditeur}

Presses universitaires de Bordeaux

\section{Référence électronique}

Marta Roca i Escoda, «Visibiliser et normaliser les familles homoparentales en Espagne. Les actions médiatiques de l'association Familles Lesbiennes et gays », Genre en séries [En ligne], 6 | 2017, mis en ligne le 01 novembre 2017, consulté le 18 février 2021. URL : http://journals.openedition.org/ges/953 DOI : https://doi.org/10.4000/ges.953

Ce document a été généré automatiquement le 18 février 2021.

\section{cc) (†) $\ominus$}

La revue Genre en séries est mise à disposition selon les termes de la Licence Creative Commons Attribution - Pas d'Utilisation Commerciale - Pas de Modification 4.0 International. 


\title{
Visibiliser et normaliser les familles homoparentales en Espagne. Les actions médiatiques de l'association Familles Lesbiennes et gays
}

\author{
Marta Roca i Escoda
}

1 Tout groupe qui entend exprimer une protestation opère une sélection au sein de l'ensemble des formes d'action qui lui sont virtuellement accessibles et qui s'inscrivent dans plusieurs espaces (juridiques, militants, professionnels, etc.). À travers les actions de l'association Familles Lesbiennes et Gays (FLG), cet article veut analyser les démarches mises en œuvre pour conférer une visibilité aux réalités familiales homoparentales afin de les doter d'un caractère de normalité. Cet objectif normatif sera au centre de leur travail de visibilisation, travail qui s'éloigne des répertoires d'action les plus utilisés par les mouvements sociaux - telles que les manifestations et autres actions de revendications publiques (Mathieu, 2008; Fillieule, 2001). Pour ce faire, cette association imposera à ses membres des formes d'organisation et d'activités spécifiques, et veillera aussi à contrôler la nature et les canaux de communication des revendications (Dupuy, Halpern, 2009).

2 Plus concrètement, nous concentrerons notre analyse sur les stratégies de visibilisation des familles homoparentales dans les médias espagnols, canaux privilégiés de l'association. Pour y accéder, la FLG met en place de grands événements, adaptés à l'accueil des médias, et donc visant une mise en scène médiatique, conçue comme un outil propice à faire passer les revendications. Pour l'association, c'est principalement à travers cette forme de présence médiatique que se réalise la visibilité des familles, d'où le soin mis à contrôler tant la scène d'apparition que le message que ces familles sont censées transmettre ${ }^{1}$. La visibilité apparaît ainsi au centre de l'activité, dont l'objectif premier revient à montrer - autant que démontrer - la chose suivante : «nous sommes des familles normales, des familles comme les autres". 
3 Nous commencerons par retracer l'histoire de l'association en mettant l'accent sur l'évolution de son travail revendicatif. Ensuite, nous analyserons ses stratégies d'action, en montrant l'importance qu'y prend la visibilité médiatique. Afin de comprendre les liens entre les mises en scène médiatiques et les opérations revendicatives, nous nous concentrerons sur deux documentaires produits par l'association: Homo baby boom et Right to Love.

4 Nos recherches antérieures ont permis d'explorer les parcours vers la reconnaissance des familles homoparentales en Espagne (Roca i Escoda, 2011). Afin de reconstruire ces processus, nous avons suivi de près le travail de mobilisation de l'association FLG. Dans ce cadre, dans un premier temps, nous avions réalisé des ethnographies historiques pour documenter les évolutions des revendications du mouvement homosexuel (Roca i Escoda, 2013). Dans un deuxième temps, nous avons enquêté sur les actions de l'association FLG (Roca i Escoda, 2017). À cette occasion, nous avons fait un travail conséquent sur les archives de la FLG, qui ont été mises à notre disposition par une des présidentes de l'association. Nous avons aussi réalisé des observations ethnographiques des groupes de travail sur l'homoparentalité, notamment pendant la préparation et le déroulement de la rencontre européenne des familles homoparentales en Catalogne (mai 2012). Plus concrètement, nous avons fait partie de l'équipe d'organisation en tant qu'expert universitaire et nous avons assisté à cette rencontre. Finalement, nous avons complété ces observations avec la réalisation d'entretiens auprès d'une vingtaine de membres actifs de ce groupe ${ }^{2}$, notamment pendant la rencontre européenne de 2012.

\section{La constitution d'un réseau de familles homoparentales et la fabrication d'une cause}

5 L'association FLG naît en $2001^{3}$, regroupant plusieurs personnes lesbiennes qui se connaissent par ailleurs et ont des enfants. Ces personnes désirent rencontrer d'autres parents, pensant que d'autres familles dans la même situation se trouvent isolées. Par l'utilisation de courriels, de proche en proche, un réseau se constitue. Une première rencontre informelle est organisée un samedi à Barcelone : environ cinquante familles, plus celles de lesbiennes que de gays, venant de diverses régions de la Catalogne, y assistent. Cette rencontre leur fait réaliser le besoin et le souhait d'un grand nombre de personnes de se réunir et échanger sur leurs expériences, comme en témoigne l'ancienne présidente, Pilar Pujol, en revenant sur l'une des premières rencontres :

Là-bas, on s'est rendu compte [elle et sa conjointe] que nous n'étions pas seules, qu'il y avait des revendications, que notre famille n'était pas du tout protégée. On a commencé à réfléchir à ce qu'on pourrait faire et on a commencé à s'organiser pour faire entendre notre voix ${ }^{4}$.

6 L'association voit donc très vite le jour ${ }^{5}$. En ce qui concerne sa structure matérielle, la FLG ne dispose pas de local. Loin de considérer cela comme une déficience, les membres y voient un avantage, puisqu'ils peuvent économiser le coût d'un loyer. Néanmoins, les activités avec les membres regroupent parfois 150 personnes. La plupart du temps, il s'agit de sorties avec les enfants, par exemple à la campagne ou dans un parc. Selon la deuxième présidente, s'offrir un local seulement pour les réunions du comité d'organisation n'aurait pas de sens, car le comité fonctionne surtout par échange d'emails et n'est constitué que de huit personnes. Si besoin, le comité se voit dans les foyers des membres. 
7 Pour ce qui est du type d'activités déployées, l'association a deux facettes. En premier lieu, elle se présente comme étant un espace de sociabilité, de rencontres et d'activités pour les familles homoparentales, ce qui engage tous les membres. En second lieu, à travers son comité, elle se mobilise à un niveau plus politique pour tout ce qui a trait à la reconnaissance des familles homoparentales, en visant l'appareil juridique, mais aussi en opérant un travail de sensibilisation dans les écoles, l'administration publique, ou les médias. Ce travail de sensibilisation sera donc souvent au centre de ses actions et se concrétisera fréquemment par une visibilité, notamment médiatique.

8 Les actions du comité ne sont pas décidées lors d'une assemblée générale regroupant tous les membres. C'est le comité qui aura le pouvoir d'action et de décision ${ }^{6}$. Le comité cherche à faire évoluer le droit afin de voir réalisés leurs droits, il s'attache à l'efficacité et prétend incarner lui-même l'idée que les actions entreprises valent pour « toutes les familles homoparentales $»^{7}$.

Dans le cas de la FLG, la cause a largement été configurée en amont de leurs actions, à travers les multiples mobilisations en faveur de la reconnaissance des couples homosexuels, en Espagne et ailleurs (Calvo, 2010 ; Paternotte, 2011). S'appuyant sur ce travail politique antérieur, dans les principales stratégies de visibilisation de l'association, on trouve : 1) tantôt des représentations scéniques de groupe, comme des images des rencontres ludiques des familles faisant partie de l'association (cf. Fig. 1) ; 2) tantôt des histoires singulières de couples qui apparaissent sous un jour " ordinaire " (cf. Fig. 2). Leur présence dans l'espace médiatique privilégie l'espace du témoin (Mehl, 2003), à travers le récit d'histoires personnelles, sentimentales et affectives, mais qui ont pourtant en charge d'illustrer une réalité collective, celles des familles homoparentales et de leur quotidien.

Figure 1

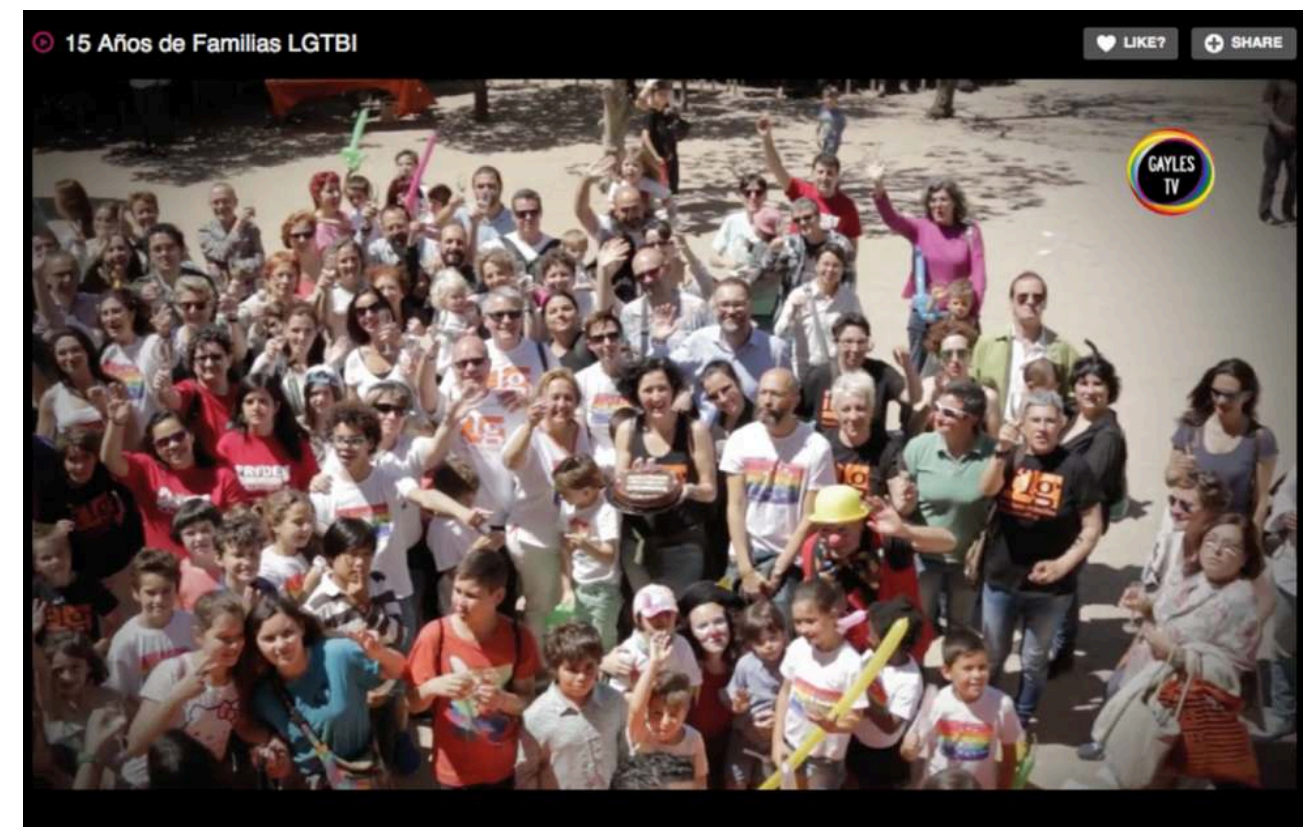

Photo FLG ( 
Figure 2. image émission TVE «Informe Semanal », 5 may 2012

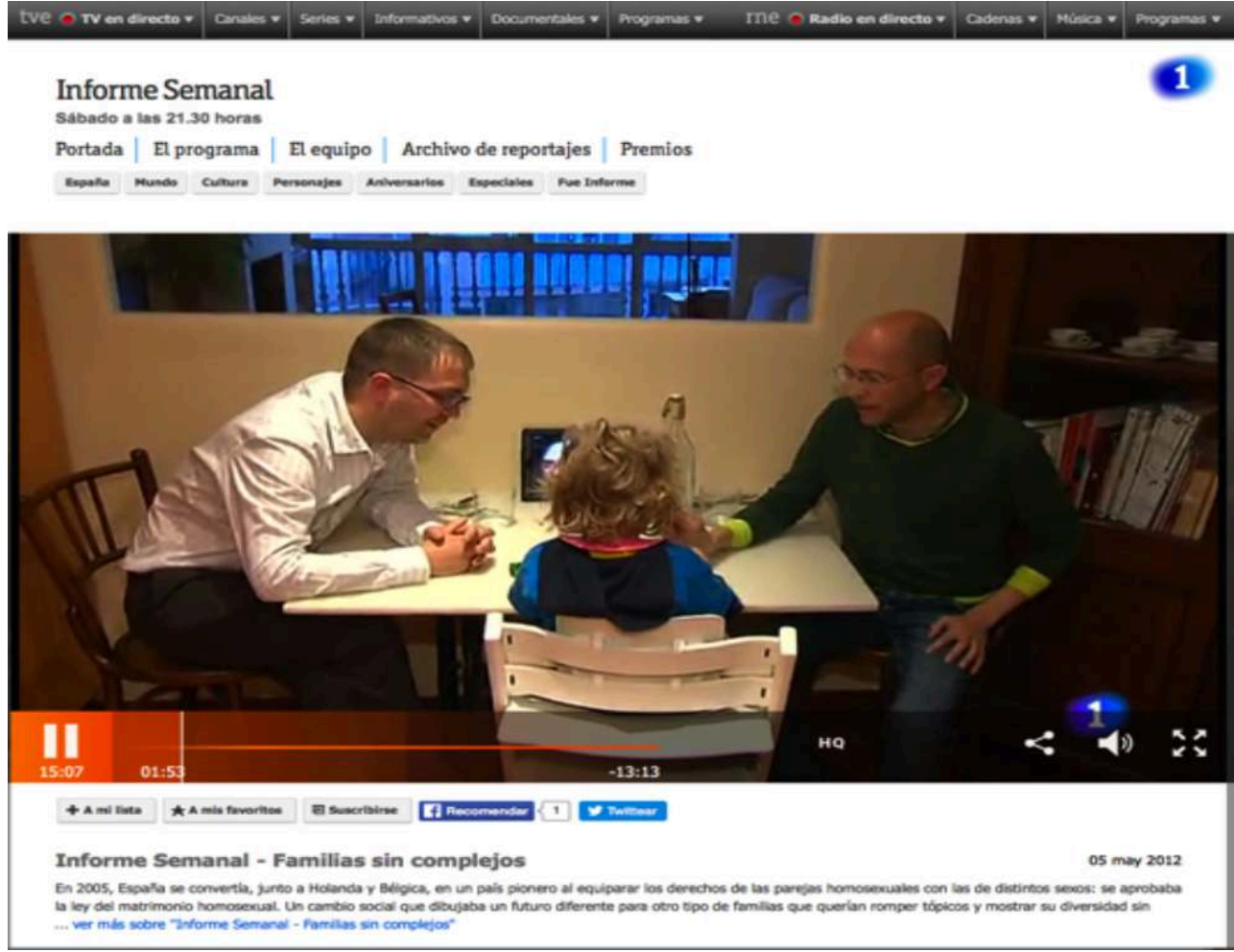

Selon sa première présidente, la création de la FLG, en raison de sa capacité à montrer l'existence des familles homoparentales saisies dans leur quotidienneté, a revêtu une grande importance au moment de la votation de la loi ouvrant le mariage aux couples homosexuels ${ }^{8}$ :

Au moment de l'approbation de la loi, on avait une association de familles, et donc ce n'était pas une chose abstraite : est-ce que nous voulons des enfants? Non, non, nous les avons déjà les enfants! Et ce n'était pas de dire : tu me fais une faveur, non. Ce sont ces enfants qui n'ont pas de droits, et ça nous donnait un argument très positif! [...] Et s'il n'y avait pas eu l'association, on n'aurait pas été en mesure de donner cette version, parce que les médias vont toujours chercher des cas très ponctuels, et ils n'auraient pas fait cette réflexion (Pilar Pujol).

11 Pour ce qui est du travail en direction de l'arène législative, la FLG a commencé avec des démarches de mise en visibilité et de sensibilisation auprès des instances politiques et administratives (Ombudsman, partis politiques, administrations, parlementaires). Dans la recherche des bons arguments, avant de se saisir et de réclamer l'application du principe d'égalité, leur démarche portait l'empreinte d'un certain pragmatisme juridique ${ }^{9}$. Elle consistait à dire que les pouvoirs publics étaient bel et bien confrontés à des «situations de fait » où des enfants avaient deux figures parentales de même sexe. Mais, en réalité, vis-à-vis de la loi, seule une figure de parent existait, de sorte que si l'une des deux disparaissait ces enfants-là n'étaient pas convenablement protégés. La réception des arguments pragmatiques formulés par la FLG s'est avérée très positive, surtout auprès des partis de gauche qui sont entrés au gouvernement catalan à partir de $2003^{10}$. La loi catalane sur l'adoption des couples homosexuels a donc été approuvée en avril 2005, deux mois avant celle du mariage homosexuel espagnol. de visibilisation des réalités familiales : la FLG se doit de montrer qu'il y a bel et bien 
des familles existantes, pour ensuite dénoncer leur manque de reconnaissance légale, et donc de protection adéquate. En ce sens, ce travail de visibilisation traverse l'ensemble de ses actions et, comme nous le verrons, implique une pédagogie spécifique, celle de la « sensibilisation » (Roca i Escoda, 2017).

\section{La composante symbolique du droit : être dans son droit et avoir le droit de se montrer}

Bien que l'un des objectifs principaux de la FLG soit la visibilité des réalités des familles homoparentales, jusqu'à la loi espagnole ouvrant le mariage aux couples homosexuels, beaucoup de membres du comité sont restés à l'écart des regards médiatiques. Selon la présidente Elisabet Vendrell, pendant les premières années d'existence de l'association et jusqu'à l'approbation de la loi catalane et espagnole, la FLG était une "association dans l'ombre " : peu de membres étaient disposés à prendre la parole et à représenter l'association dans les médias. E. Vendrell ira jusqu'à nous confier que, dans son couple, si sa compagne avait fait son « coming out », elle, non.

Pour les membres actifs de l'association, au début, la sphère juridique et ses prolongements administratifs primaient, en jouant comme l'opérateur et le médium de leur aspiration à être reconnus en tant que "famille", reconnaissance qui leur permettrait de pouvoir réaffirmer des liens de parenté avec leurs enfants respectifs au sein de couples gays et lesbiens (Roca i Escoda, 2010).

Le droit a donc une composante symbolique et c'est en raison de cette dimension qu'il a permis de conférer une visibilité et une légitimité aux acteurs dans la poursuite de la cause de l'association FLG. Aux yeux de ses membres, et aussi de leur entourage, c'est par le moyen du droit que leur cause est apparue de plus en plus légitime. En leur donnant cette force que leur confère l'assurance d'être dans leurs droits, et d'agir pour la réalisation du droit, la loi était ainsi pourvue d'effets agentifs (Roca i Escoda, 2011). Le récit de la présidente de la FLG à propos de la loi sur le «mariage homosexuel » va dans ce sens :

Et ça a fait comme un déclic pour quelques familles, elles se sont dit : « nous devons nous montrer ». Pourquoi ? Parce que dans nos cercles les plus familiers, dans le quartier, à l'école de nos enfants, les gens ont connu notre réalité familiale. Personne ne nous a refusés, personne n'a causé de problèmes, et ça nous a aidés (Elisabet Vendrell).

Étant donné le type d'actions envisagées et la nature de la problématisation mise en œuvre (la reconnaissance des familles homoparentales), comme nous l'avons souligné, la FLG s'est engagée dans un gros travail de visibilisation, avec une claire intention pédagogique : il s'agissait de révéler une réalité existante et de la rendre normale. Son but était donc l'acceptation sociale et publique de cette réalité familiale.

Ce travail de visibilisation va ici de pair avec un désir de normalisation (i.e. « rendre normal»). Selon Axel Honneth, l'invisibilisation doit être comprise comme la production d'une non-existence, au sens social du terme (Honneth, $2006: 225$ ), mais, paradoxalement, elle renvoie aussi à une visibilisation (politique/médiatique) qui contribue à "faire exister le groupe ailleurs que dans son contexte immédiat d'existence » (Voirol, $2005:$ 18). Les propos d'un membre de l'association vont dans ce sens : 
Nous utilisons beaucoup la phrase suivante : « ce qu'on ne veut pas n'existe pas ». Et pourtant, si on n'existe pas, il n'y a pas besoin de lois pour nous protéger. Il n'y a personne qui se plaint, qui se sent discriminé du moment qu'on n'existe pas. En quelque façon, on doit se rendre visible (Membre FLG).

Et c'est bien ce que les actions de la FLG se sont attachées à viser, et cela depuis sa constitution en tant qu'association. Pour ses membres, se doter d'une existence collective et veiller à la faire reconnaître, c'est aussi affirmer une normalité. Cette affirmation passera souvent par la mise en scène médiatique d'une série de témoins singuliers qui seront toujours reliés à une cause collective et générale: celle de la reconnaissance des familles homosexuelles.

Donner une image d'une très grande normalité, qui était là mais qu'on ne voyait pas [...] Alors le moyen : regarde, je suis en train d'attendre ma fille à la sortie de l'école [...]. Je crois que le truc c'était d'être là au moment qu'il fallait et de voir que ce n'était pas un cas isolé, mais qu'il y avait beaucoup de familles qui étaient associées et qui avaient ces problèmes pratiques vis-à-vis de leurs enfants (avocate, membre de l'association).

19 En effet, dans les actions autour de cette lutte pour la reconnaissance des familles homoparentales, nous constatons deux ressorts ${ }^{11}$ dans la volonté de «rendre normal » et d'«affirmer une normalité ». D'une part, il s'agit de pouvoir montrer que ces familles sont conformes au type le plus fréquent, dans le sens de courant, en insistant sur les traits d'une quotidienneté (i.e banalité). Et d'autre part, de par cette ordinarité, il faut pouvoir se débarrasser de tout attribut pathologique (les préjugés concernant l'homosexualité). Pour la FLG, il s'agit donc de rentrer dans la norme de ce qui est attendu d'une famille, en renforçant son caractère normatif, « c'est-à-dire à cela qu'il n'est pas seulement une norme signifiant une certaine exigence, mais entraînant, ou tendant à entraîner le comportement conforme à cette exigence " (Legrand, 2007 : 4).

Pour ce faire, le comité a logiquement cherché à se doter d'une identité positive et consensuelle. En ce sens, l'association a fait réaliser un film-documentaire en 2008, Homo Baby Boom (Fig. 3), vendu sous forme de DVD ${ }^{12}$. Celui-ci est accompagné d'outils pédagogiques pour être visionné dans les écoles. Ce documentaire vise à sensibiliser les familles, les enfants et les enseignants, dans et dès le cadre scolaire. 
Figure 3. image documentaire " Homo Baby Boom »

\section{Homo Baby Boom families de lesbianes i gais}

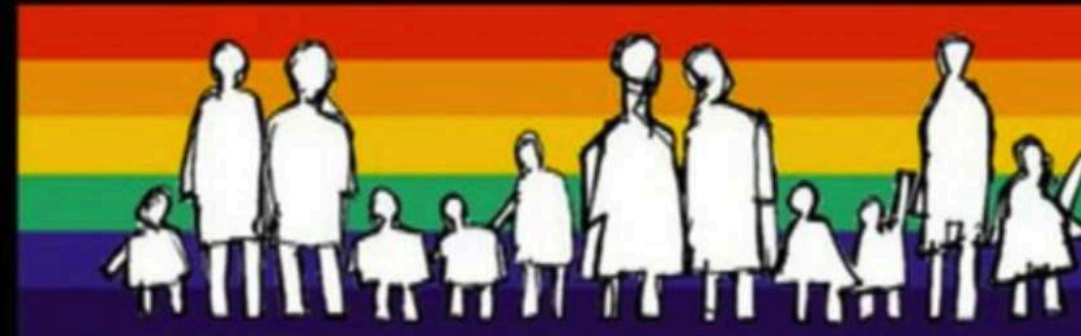

Un documental d'Anna Boluda i l'Associació de families Lesbianes i Gais www.homobabyboom.com

Ce documentaire, produit par la FLG, présente des moments de la vie quotidienne de six familles homoparentales de Catalogne et de Valence. Trois familles de mères lesbiennes et trois familles de pères gays expliquent comment ils ont eu leurs enfants. Ils témoignent des réactions rencontrées de la part de leurs familles et de leur environnement. Ils reviennent également sur l'incidence que les changements légaux de 2005 ont eue sur leurs vies.

Plus concrètement, le documentaire - qui dure 27 minutes - commence par rendre compte de la préoccupation majeure des familles homoparentales, l'acceptation sociale, et de leur plus grand défi, la visibilité. Avec un cumul de scènes de la vie quotidienne, il montre comment ces familles sont «des familles normales, des familles comme les autres». Pour ce faire, il est mis en avant un fait universel: «l'amour pour nos enfants ».

Une série de plans filmés des activités les plus routinières du quotidien de ces six familles constitue le cœur du documentaire : se brosser les dents, peigner les enfants avant de les amener à l'école, jouer ensemble, lire, faire à manger, manger. Ces situations ordinaires d'une extrême banalité sont filmées en plan rapproché et montrent toutes le travail du care, l'affection et l'amour portés aux enfants (Fig. 4). 
Figure 4. Image du documentaire «Homo Baby Boom »

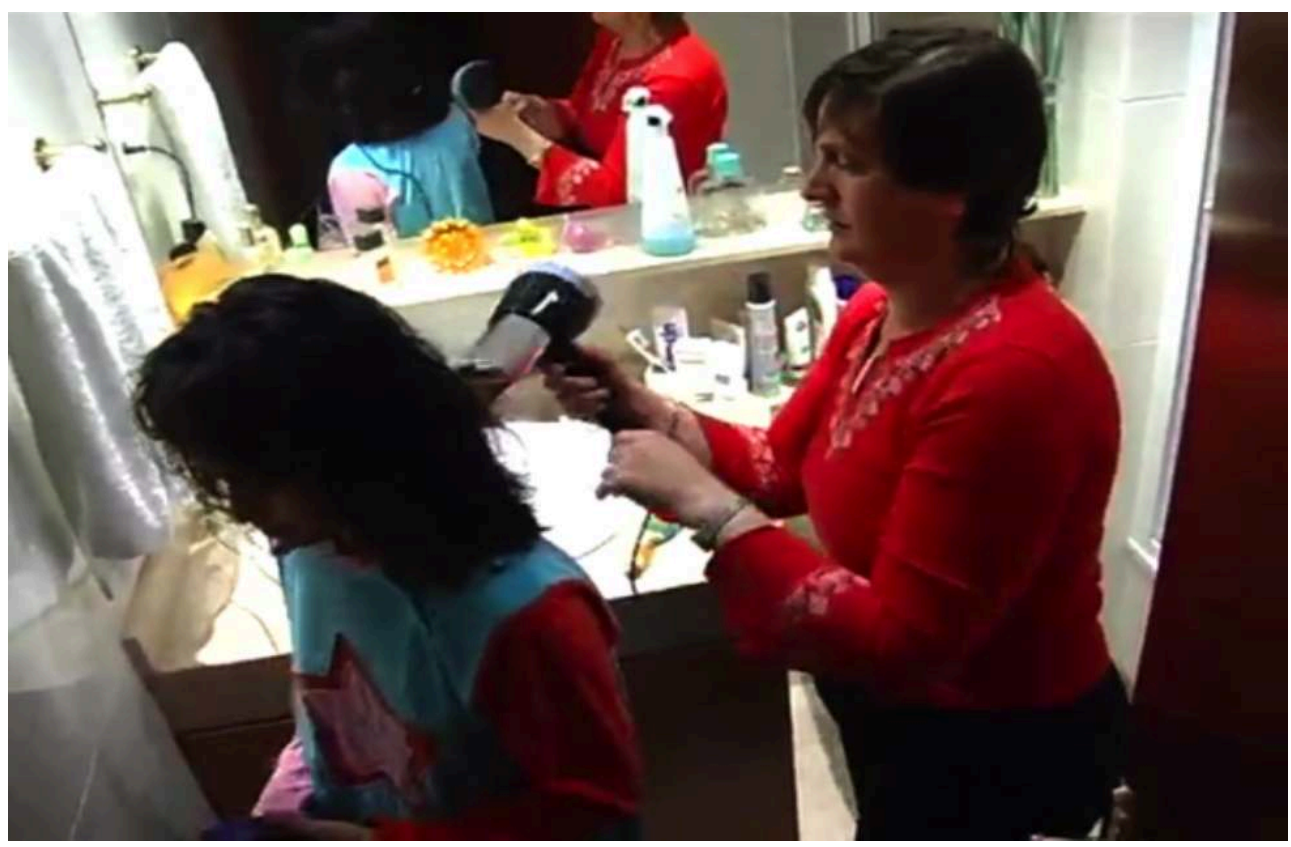

La deuxième partie du documentaire se focalise sur les avancées légales pour montrer comment elles ont contribué à la normalisation de ces familles en Espagne. À cette fin, les parents témoignent de leur parcours de parentalité (Roca i Escoda, 2016), des réactions de leur entourage, de la famille et de l'école. Les enfants seront également portés au témoignage pour expliquer leur vécu tout en affirmant une normalité : "Nous sommes des enfants comme les autres, la seule différence est que nous avons deux papas, deux mamans ». Toujours dans un but pédagogique, on apprend aussi ce qu'un ensemble d'études disent de ces réalités familiales, afin de contrer les peurs et de lutter contre les préjugés.

25 Mais comment cette normalité a-t-elle été expressément travaillée afin d'être montrée dans ce documentaire? Une adhérente de l'association nous explique ainsi que les membres du comité cherchaient les bons arguments et les meilleures manières de présenter les choses. Selon elle, le comité décida de ne pas aller chercher des cas négatifs, comme : «ma partenaire m'a quitté, et empêche les enfants de me voir ». Ils ont décidé de ne pas recourir au registre tragique ou dramatique. Au contraire, ce qu'ils ont voulu montrer ce sont des mères et pères qui habitent ensemble dans des maisons familiales, des parents qui travaillent dans les écoles et dans d'autres métiers « exemplaires », comme médecin, avocat, etc.

Un membre de la FLG nous confie que le comité a choisi des témoignages de couples homosexuels très "standards », en excluant volontairement des couples de lesbiennes "très masculines", des couples de gays très "maniérés " ou encore des couples de trans*. Cette volonté de reconnaissance va de pair avec une standardisation genrée (hétéronormative) des familles homoparentales. Comme le souligne Lilian Mathieu, " un groupe qui lutte pour sa reconnaissance et pour la disparition des mesures discriminatoires ou des attitudes et discours stigmatisants est pris dans une tension parfois délicate à gérer. Doit-il exiger qu'on l'accepte tel qu'il est, avec les traits distinctifs qui fondent sa stigmatisation, ou doit-il donner des gages de sa capacité à 
s'intégrer sans problème dans la majorité - quitte au besoin de remodeler son identité pour la rendre plus 'acceptable' ?» (Mathieu, $2004: 142$ ).

Dans le langage public de son action, le groupe, tout à sa quête d'acceptabilité, mettra ainsi l'accent sur un vocabulaire généralisant susceptible de le rapprocher de la «majorité » de la population: d'où ce choix de se présenter comme des familles très ordinaires, voire exemplaires dans leur banalité. Comme le souligne Luc Boltanski, dans le travail de dénonciation, une affaire est exemplaire «lorsqu'elle mérite d'être portée à l'ordre de la dénonciation publique, non pour elle-même, mais dans la mesure seulement où elle constitue un cas remarquable à l'intérieur d'une série caractérisée par son meilleur exemple " (Boltanski, 1990 : 306). C'est notamment le cas de l'un des témoignages, celui d'un couple gay qui adopte un enfant trisomique et qui témoigne d'une envie de donner de l'amour à cet enfant qui ne serait pas spontanément désiré par un couple standard. Face à un tel témoignage, le spectateur ne peut que les admirer dans leur démarche de devenir parent (Fig. 5).

Voici comment le couple en témoigne: "Nous avons parlé de l'envie d'adopter un enfant ayant des problèmes. Là, notre difficulté d'être parents, nous a sensibilisés en ce sens ». La dimension émotionnelle qui traverse tout le documentaire est renforcée par le témoignage de ce couple exemplaire, notamment lorsqu'il relate le moment où ils ont su qu'ils pouvaient adopter un enfant :

On nous appelle un lundi à la maison, j'étais seul, Jacint travaillait, et on m'appelle : Carles, demain matin vous devez descendre à Barcelone à telle heure car vous avez une adoption. Et je raccroche le téléphone..., et je commence à trembler et à pleurer, ici seul sans pouvoir le dire à personne...

- la première sensation... On ne le croit pas vraiment, et quand tu l'as (l'enfant) et tu vois qu'il est avec toi, et qu'il sera toujours avec toi ! oh!!!

Figure 5. Extrait documentaire « Homo Baby Boom »

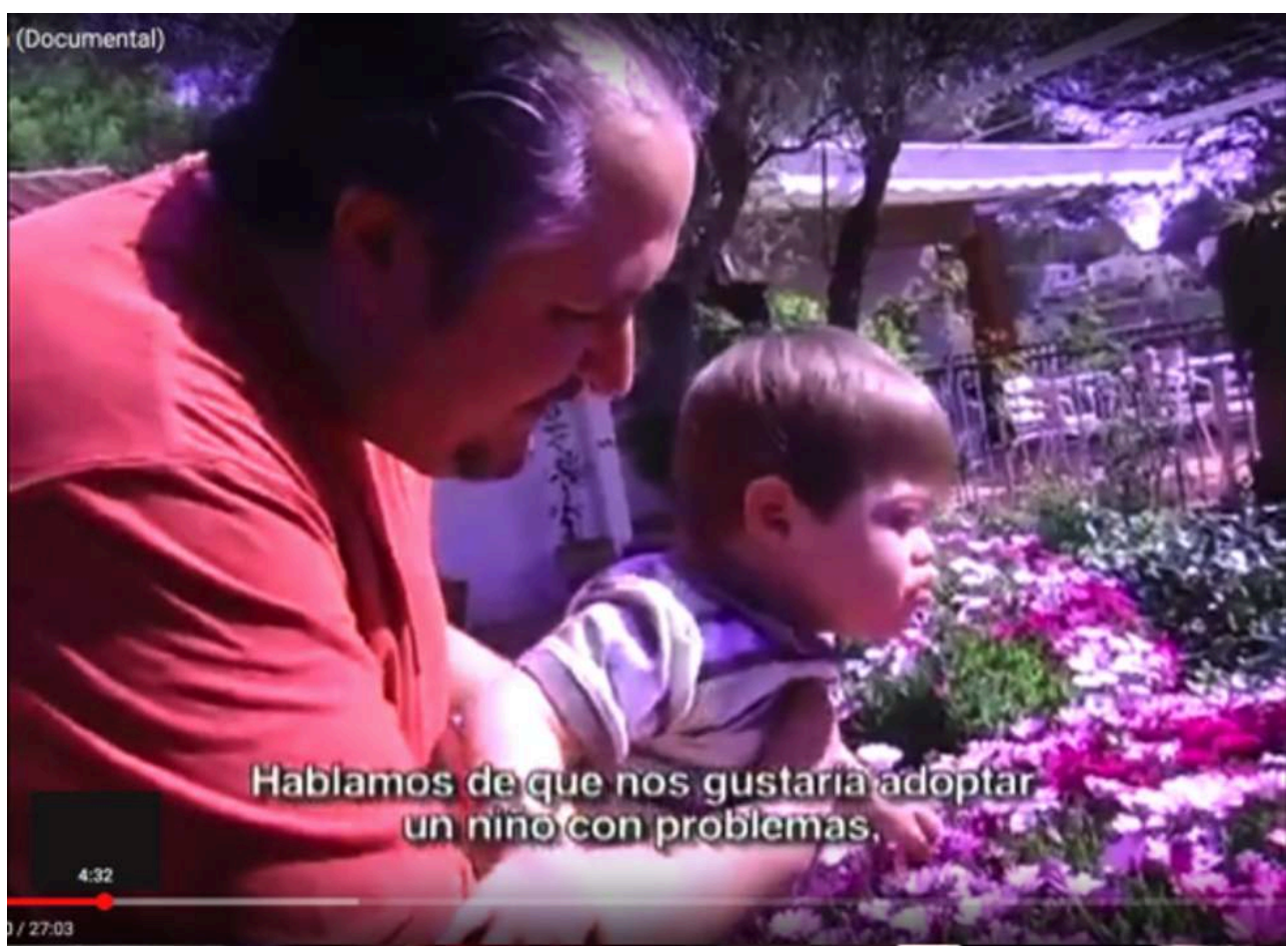



soulignent les dimensions éthiques et morales. Comme le souligne Déchaux, "les émotions peuvent être le produit d"effets de cadrage', c'est-à-dire résulter de la présence dans l'environnement social de scripts ou de grammaires affectives disponibles et légitimes » (Déchaux, 2015). Dans ce sens, les couples choisis attestent en personne de ce que tout un chacun attend d'une famille exemplaire, au-delà de sa composition atypique. Face à la caméra, avec leurs enfants respectifs, deux femmes ou deux hommes deviennent deux parents et constituent ainsi une famille aux yeux de spectateurs/trices, qui sont ainsi invité.e.s à ressentir des émotions positives, entrant en résonnance avec les affects et sentiments que manifestent à l'écran les personnages $\mathrm{du}$ documentaire filmique. Comme le soulignent Malbois et Kaufmann, «l'expression publique de l'émotion doit répondre à une condition de recevabilité ou de crédibilité : l'émotion revendiquée doit être ajustée au bien qui, selon le locuteur, a été affecté " (Malbois et Kaufmann, 2014: 100-101). Dans notre cas, le bien réclamé est la famille dans sa définition courante et en son existence idéale : des adultes qui prennent en charge des enfants, les éduquent et les aiment. Notons bien qu'il y a donc une volonté normative très forte dans la démarche d'être reconnus comme famille. Cette volonté s'accompagne d'un processus de normalisation : « nous sommes des familles comme les autres »(membre FLG).

\section{Changements dans le travail de visibilisation}

Dès sa naissance en 2001, l'association a fait un gros travail de "marketing " pour se faire connaître. En 2004, une année avant l'ouverture du mariage aux couples homosexuels, un des objectifs principaux fixés par le comité est la visibilité, en soulignant le besoin de membres qui apparaissent dans ces médias, mais aussi en soignant la présentation de la page web de l'association. La conception du site internet de la FLG sera confiée à des professionnels et les pages feront l'objet d'une mise à jour quotidienne. Un budget considérable lui est alloué ( $40 \%$ du budget de l'association est destiné à l'infrastructure web et aux salaires, notamment du webmaster et du chargé de communication $)^{13}$.

31 Après l'adoption de la loi accordant le mariage aux couples homosexuels en 2005, les actions de la FLG vont donc se focaliser sur un travail en direction de et avec les médias, toujours selon cette double visée: "Continuer à travailler pour la sensibilisation sociale. Continuer à travailler pour la visibilité des familles lesbiennes et homosexuelles dans les médias » (rapport d'activité 2008). Le même rapport d'activité souligne ainsi que la FLG a été vue ou entendue dans plus de cinquante médias en une année ${ }^{14}$.

ses opérations de visibilisation, la FLG continue à veiller à se présenter sous un aspect de normalité, en se montrant sous le jour de familles « comme les autres », avec toutes les qualités, mais aussi avec tous les problèmes quotidiens liés à l'éducation des enfants. Pour autant, la composante identitaire est aussi présente, et, au fil du temps, elle se trouve même de plus en plus assumée et problématisée : «c'est précisément parce qu'on est différents (famille homoparentale) que nos enfants et nous-mêmes sommes discriminés » (page web FLG).

Le deuxième documentaire, Right to love, produit par la FLG à l'occasion de la deuxième rencontre européenne des familles LGBT qu'elle organisa en mai 2012 - et réalisé par la 
même journaliste que Homo Baby Boom -, atteste de cette évolution de l'affirmation identitaire mais aussi revendicative (Fig. 6).

Figure 6. Affiche documentaire « Right 2 love»

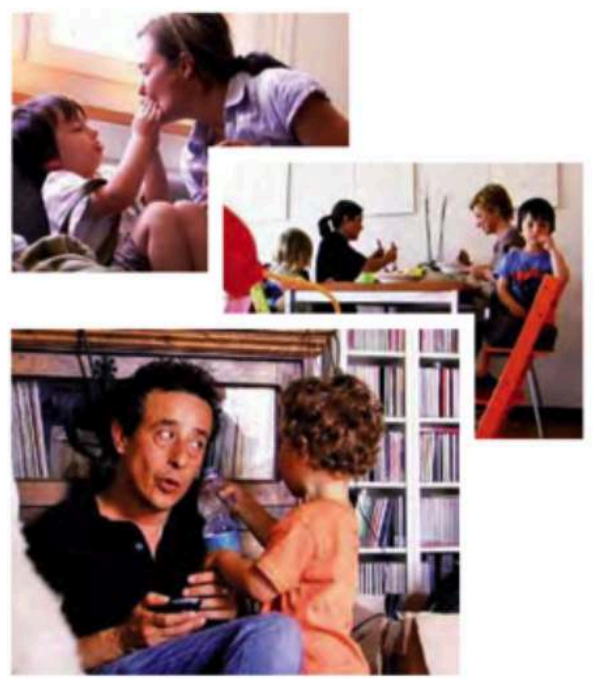

\section{Righ§2 love}

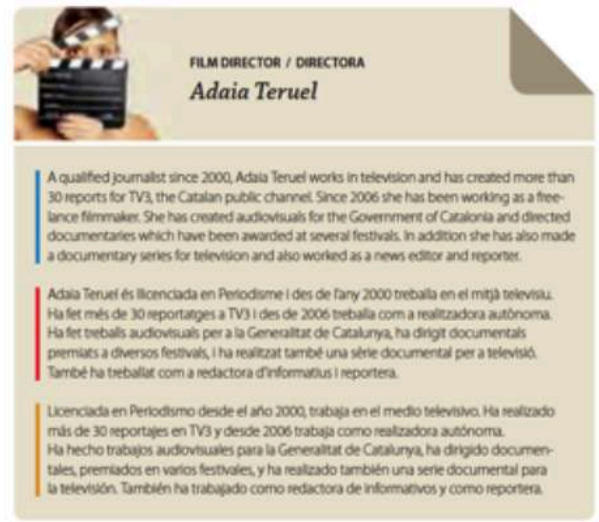

D'une part, les couples sont moins standards, comme l'atteste le cas d'un couple comprenant une personne transsexuelle (Fig. 7). D'autre part, leurs revendications intègrent des aspects nouveaux, comme la gestation pour autrui. Autre nouveauté, la visée revendicative de la FLG s'agrandit, car elle prétend toucher l'Europe. Le documentaire se compose ainsi de familles de nationalités européennes différentes.

Figure 7. Extrait du documentaire « Right 2 love »

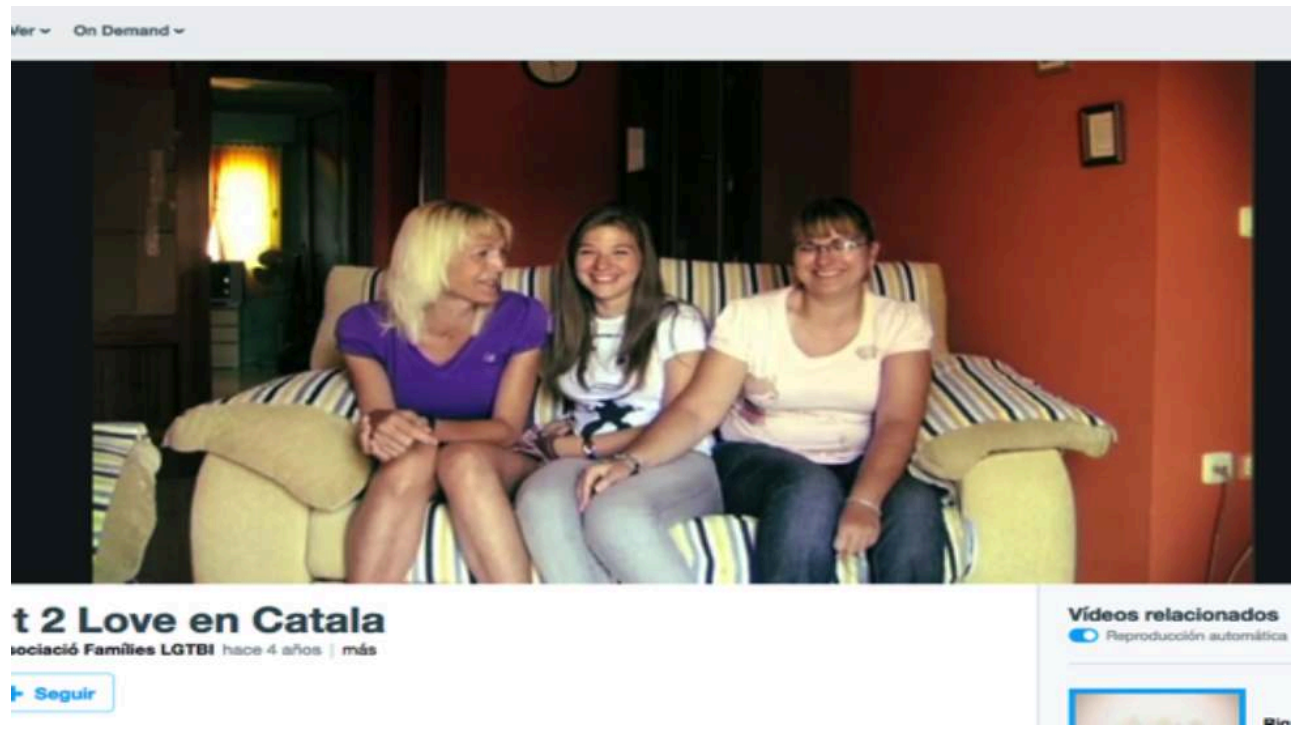

Right to love donne la parole à sept familles de sept régions de l'Europe, qui diffèrent quant au degré de reconnaissance institutionnelle des familles homoparentales. C'est le cas d'un couple de femmes néerlandaises, le premier couple à s'être marié après la loi de 2001. En contraste, le documentaire donne également la parole à un couple gay italien et un à couple lesbien grec, l'un et l'autre ne bénéficiant d'aucune 
reconnaissance ou protection. Le schéma narratif utilisé est similaire à celui du premier documentaire : sous la forme d'entretiens biographiques, les couples témoignent de leur vie conjugale jusqu'à dénoncer les difficultés qu'ils rencontrent au quotidien. La scène filmée est composée d'un premier plan sur le couple qui témoigne, ensuite viennent des images de leur vie quotidienne avec leurs enfants : une mère qui aide à faire les devoirs à son fils; un couple de femmes qui se baigne avec leur fils; un père gay qui joue avec son enfant, une mère lesbienne qui prépare le goûter pour ses deux enfants, etc.

Les récits de ces couples ont une logique commune. En premier lieu, ils témoignent de leur histoire d'amour, de leur rencontre, en second lieu, ils retracent le parcours de leur parentalité. La pédagogie de ces récits est telle que ces paroles pourraient constituer des extraits d'entretiens que l'on trouve dans la littérature scientifique concernant l'homoparentalité et la question homosexuelle: le désir d'enfant des couples gays, étudié par Martine Gross (2009) et Jérôme Courduriès (2011) ; les parcours de la parentalité avec les diverses modalités que cela peut prendre, étudiés par Anne Cadoret (2011); des cas de familles recomposées (Domingo, 2009); d'adoption (Fine, 1998); de PMA avec donneur connu et donneur anonyme (Mehl, 2011). Il est ainsi question du difficile processus de la GPA (Gross et Mehl, 2011, Courduriès, 2016), de la paternité gay (Gross, 2012), de la maternité lesbienne (Decoutures, 2010), et aussi de la parenté transgenre (Hérault, 2014) ${ }^{15}$.

À ces couples témoins s'ajoutent les témoignages des enfants. Comme cela avait aussi été le cas dans le premier documentaire, ces enfants disent la normalité familiale dans laquelle ils vivent, manifestant l'amour et l'affection qu'ils reçoivent de leurs parents. Enfin, deux personnalités apparaissent dans la dernière partie du documentaire, une personnalité politique et une personnalité scientifique: Raúl Romea, vice-président intergroupe des droits LGBT au Parlement européen, et Robert Wintemutte, professeur des droits humains au King's College, évoquent la lutte pour les droits civiques et humains. La question des droits sera au centre de cette dernière partie du documentaire, qui souligne les dix ans de lutte de la FLG et les buts que l'association s'est fixés : la visibilité sociale "afin d'acquérir une intégration à cent pour cent", comme l'énonce sa présidente dans l'ultime séquence du documentaire.

Right to love est en continuité avec Homo baby boom. Il s'agit toujours de montrer cette réalité familiale comme une réalité des plus banales, exemplifiant les attributs que tout un chacun peut accorder à l'idéal-type de la famille: le soin, l'affection, l'amour, l'éducation. Autre trait commun, le couple gay choisi à dessein comme couple exemplaire dans Homo baby boom, apparaît à nouveau, avec un nouvel enfant, une petite fille de deux ans. Ce couple refera le même récit émouvant de l'adoption de leur premier enfant, trisomique.

La toute fin du documentaire constitue une scène filmée où quelques membres de l'association et leurs enfants invitent le spectateur à la rencontre européenne des familles homoparentales que celle-ci organise en mai 2012. 


\section{Une mise en scène maîtrisée : la rencontre européenne des familles homoparentales 2012}

On l'a vu, se présenter publiquement est une activité essentielle dans les démarches de revendication de la FLG. Dans la mise en scène médiatique de la mobilisation (Fillieule, 2014), la FLG ira jusqu'à engager une agence de communication pour la préparation des communiqués de presse et des diverses présentations dans les médias et sur d'autres scènes publiques lorsque les lois catalane et espagnole seront approuvées, mais aussi pour soutenir la visibilité de la rencontre européenne de familles homoparentales, organisée par la FLG en mai 2012 en Catalogne (à Lloret de Mar). Avec ces démarches, l'association se dote d'une « compétence médiatique » (Mathieu, 2012).

Figure 8. Affiche de la rencontre européenne familles LGBT, 2012

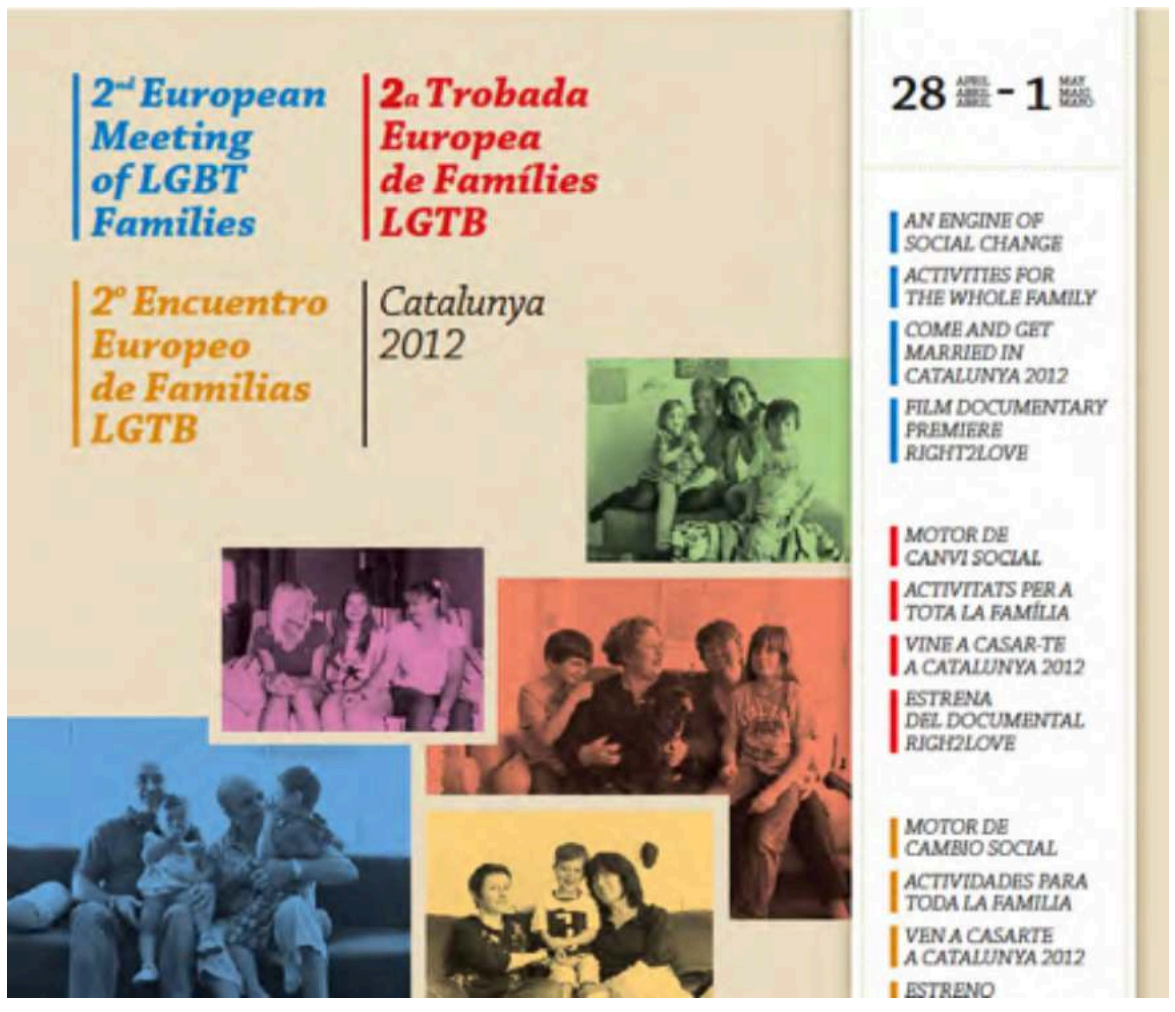

Cette rencontre représente le point culminant du travail visant à augmenter la couverture médiatique de leur mobilisation (Fig. 8). Les médias y deviendront de vrais partenaires, au point que la FLG construira un réseau médiatique qu'elle veillera à contrôler par la suite ${ }^{16}$. Lors de cette rencontre, quasiment la totalité des chaînes télévisées et des radios publiques espagnoles (et une grande partie des chaînes privées), les plus grands journaux et la presse locale, ont été recensés et publiées dans les rapports d'activité de l'association, tout cela sans compter les nouvelles diffusées par internet.

Cette volonté de privilégier la communication médiatique, tout en se dotant d'une intelligence réflexive sur les enjeux d'une communication maîtrisée, est inscrite dans les objectifs de l'association, comme en atteste le rapport d'activité de 2015 :

La visibilité, l'un de nos principaux objectifs, et la présence de familles qui représentent la FLG dans la presse est très importante pour faire connaître notre 
réalité et pouvoir réaliser des synergies avec le reste de la population. En outre, ces interventions sont également très utiles pour faire entendre nos revendications. Nous avons encore un long chemin à parcourir parce que nos droits ne sont pas garantis. Nous devons continuer à veiller à ce qu'ils s'accomplissent (rapport d'activité 2015, archives FLG).

(11

La plupart des relais de l'événement ont été configurés par les médias encore et toujours à travers des témoignages de couples qui parlent de leur quotidien, mais aussi des problèmes qu'ils rencontrent. Ce mode de traitement des enjeux collectifs par une mise en série de cas individuels n'est pas seulement un raccourci des médias, c'est en fait le mode privilégié par la FLG dans son travail de visibilisation des problèmes affectant les familles gays et lesbiennes.

44 Les couples témoins n'ont pas été choisis au hasard, il fallait qu'ils puissent se prêter au modelage d'une identité publique stable (Barbot, 1999), stratégiquement visée par la FLG. Ce sont ainsi presque toujours les mêmes couples qui ont été interviewés par la presse, notamment le couple formé par la deuxième présidente et sa compagne, et un couple gay composé d'un professeur de sociologie, actuel vice-président de l'association, et d'un médecin, directeur de l'unité de pédiatrie d'un hôpital public catalan. Ces personnes, fonctionnant comme réseau d'opérateurs (Neveu, 1999) peuvent garantir l'apport de diverses ressources qui les habilitent à parler au nom de toutes les familles homoparentales. Elles ont deux types de légitimité, si l'on suit les analyses de Lillian Mathieu: une légitimité interne, car ces couples incarnent parfaitement les caractéristiques du groupe minoritaire ; et une légitimité externe, car ces couples possèdent « les propriétés sociales reconnues par leurs pairs ", mais aussi par un plus large public (Mathieu, 2001) ${ }^{17}$.

On atteste donc d'un "processus de professionnalisation des sources » avec une incorporation d'un savoir-faire en matière de gestion des médias, mais aussi d'anticipation sur les critères de newsworthyness par les acteurs des mouvements sociaux (Neveu, 1999 : 42), c'est-à-dire, les critères que les professionnels des médias considèrent pertinents et légitimes. 
Figure 9. Image du téléjournal de la télévision catalane (TV3)

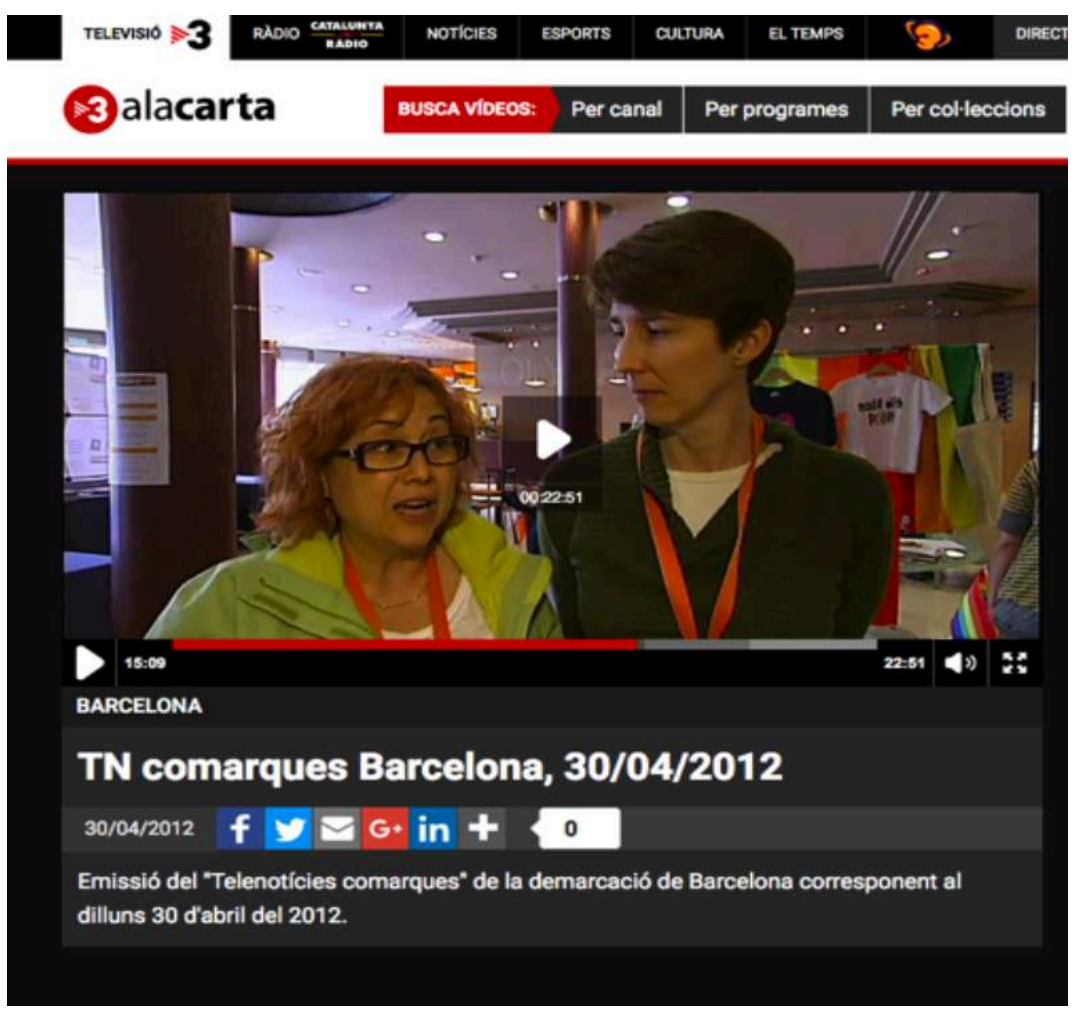

La rencontre de familles LGBT de mai 2012 regroupa plus de 800 familles, venues de divers pays européens. Trois jours au programme intense se sont déroulés dans un hôtel situé en bord de mer d'une région touristique catalane. Les familles logeaient dans cet hôtel, et avaient accès à toutes les activités proposées par la FLG : conférences, ateliers, activités en famille, soirées festives. Une crèche avait été organisée pour prendre en charge des enfants de tous âges pendant toute la journée.

Outre le fait d'avoir créé cet espace de rencontre, l'évènement était conçu comme une mise en scène à destination des médias, donnant visibilité à ces familles. Le hall de l'hôtel était aménagé pour recevoir les journalistes, un espace ad hoc servait aux entretiens avec la presse, tandis qu'un autre était destiné à la production de photographies ou d'interviews filmées (Fig. 9 et 10). L'association engagera à cet effet deux attachés de presse pour aider à couvrir médiatiquement l'événement. Dans son rapport d'activité, la FLG a comptabilisé 122 apparitions liées à la rencontre dans les médias les plus divers, locaux, nationaux et internationaux. 
Figure 10. Image du téléjournal de la télévision catalane (TV3)

\begin{tabular}{|c|c|c|c|c|c|}
\hline 3 RhDio $\frac{\text { caraunury }}{\text { Rabio }}$ & NOTICIES & ESPORTIS & cutruR & EL. TEMPS & (s) \\
\hline irta & BUSCA VIDEOS & Per cana & & Per programes & Per col-leccions \\
\hline
\end{tabular}

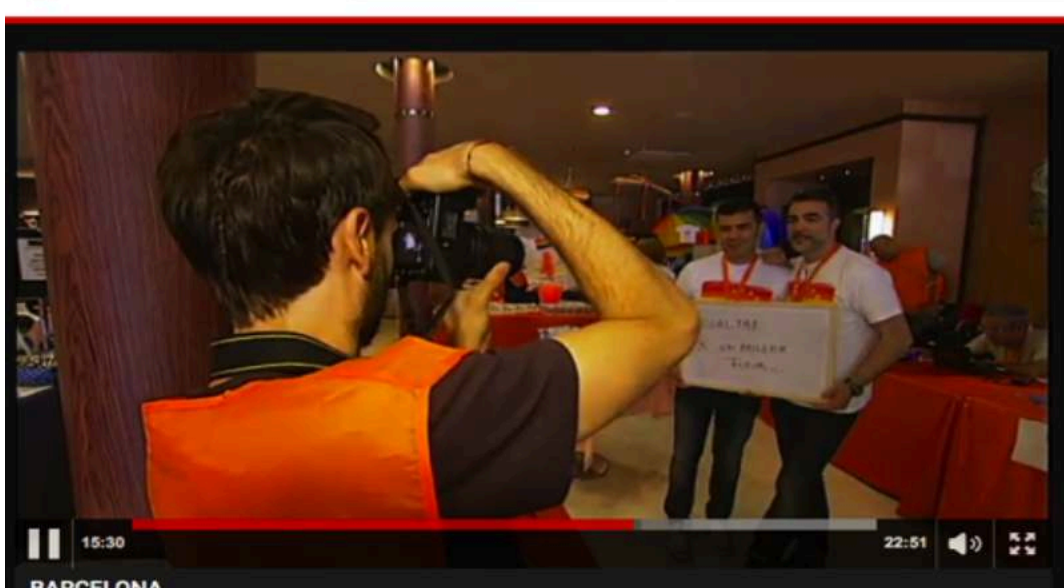

BARCELONA

TN comarques Barcelona, 30/04/2012

$30 / 04 / 2012$ f $y=G+$ in +0

Emissió del "Telenotícies comarques" de la demarcació de Barcelona corresponent al dilluns 30 d'abril del 2012.

48 La FLG impulse la création de moments et situations ad hoc pour partager les émotions, comme la cérémonie de mariage collectif fictif de douze couples célébré par le maire du village, renforçant par ailleurs cette mise en scène à destination des médias (Fig. 11 et 12). Ce moment de la rencontre a d'ailleurs été montré par la plupart des médias qui ont couvert l'événement.

Figure 11

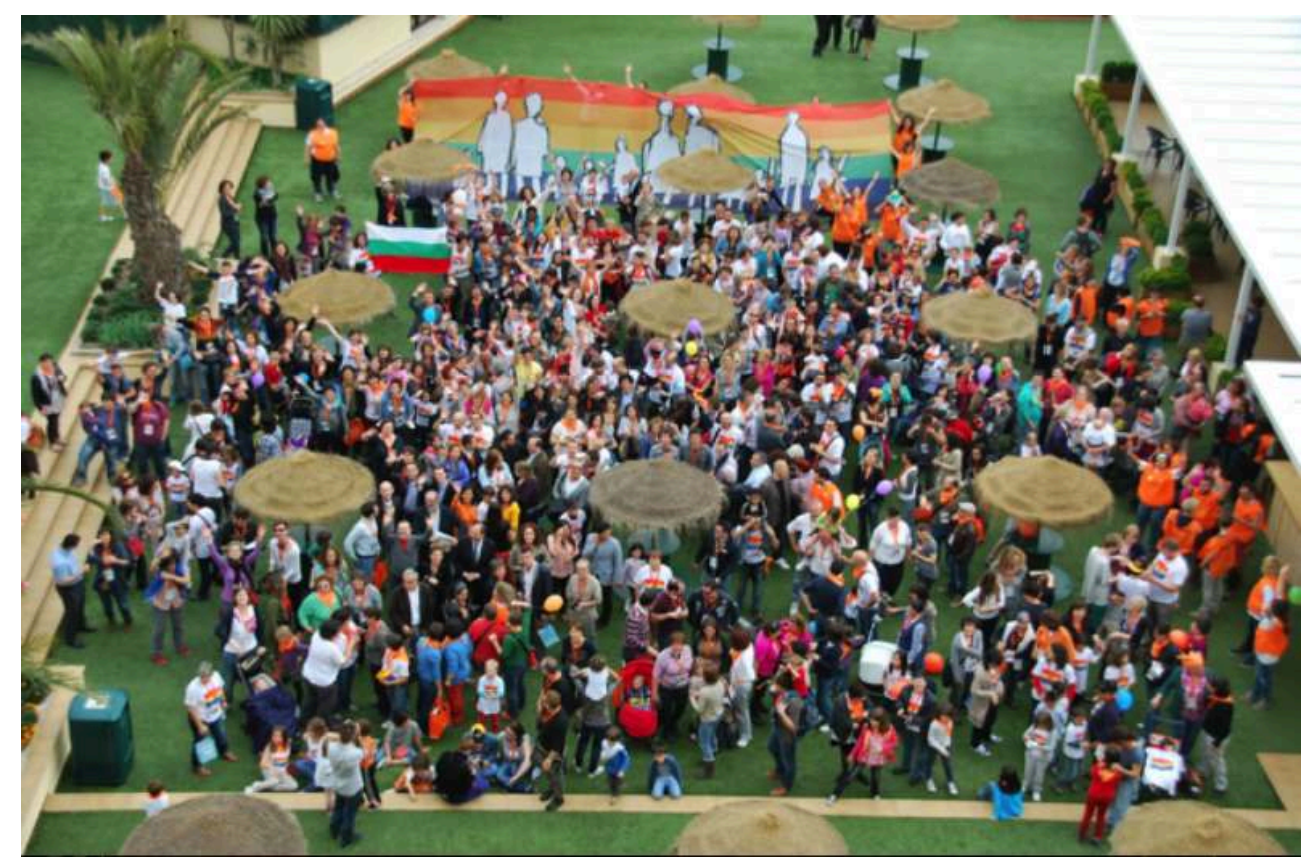

Photo FLG @ 
Figure 12

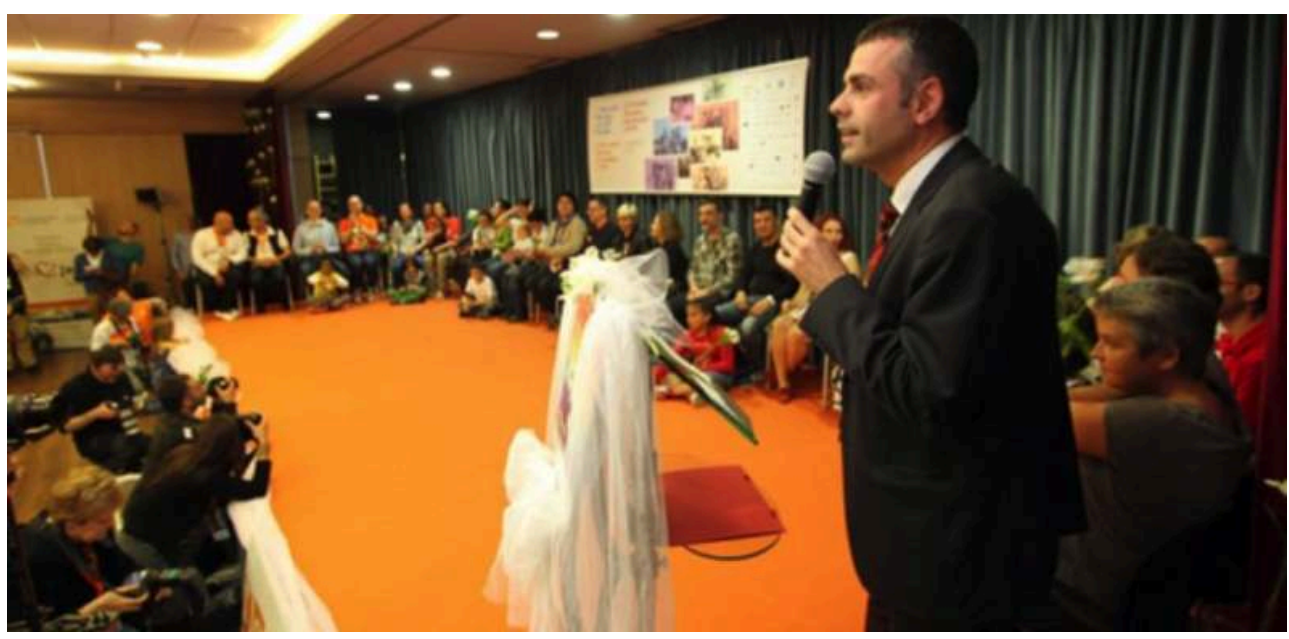

Photo FLG ๔

\section{Conclusion}

L'étude du travail militant de la FLG montre l'utilisation des médias comme véhicules et canalisation d'une revendication dans l'espace public. Les médias ne sont pas seulement des destinataires de la communication de l'association, mais deviennent de véritables relais de cette dernière. Comme le souligne Neveu, «Une des tendances des mouvements sociaux contemporains - spécifiquement dans les pays dotés d'un 'espace public' marqué par un minimum d'ouverture et de pluralisme - réside dans l'attention croissante que leurs organisateurs dédient à une gestion réfléchie de leur rapport aux médias» (Neveu, 2010 : 246). Au travers d'exemples de diverses actions médiatiques, nous avons pu montrer ce rapport réflexif que ses membres-experts ont à l'endroit du travail médiatique. Mais pour le cas qui nous occupe, il ne s'agit pas d'une "dépendance accrue des mobilisations à l'égard de médias publics ou privés qu'ils ne contrôlent pas pour faire passer leur message vers le public le plus étendu » (Neveu, Ibid); car la FLG maîtrise la gestion du travail médiatique, comme l'atteste la mise en scène de la rencontre européenne en 2012. En effet, les modalités de monstration dans les médias vont d'une mise en scène très maîtrisée, un hall d'hôtel aménagé à cet effet, et d'une apparition médiatique avec des témoins choisis à dessein, tels que les leaders associatifs.

50 Au travers de cette étude de cas, nous avons aussi voulu montrer que l'appareil juridique, notamment l'ouverture de nouveaux droits, donne autorité et légitimité aux membres de la FLG ; s'ils persistent dans leur démarche, c'est précisément parce qu'ils estiment en avoir le droit et s'appuient sur la force que leur confère ce qu'ils tiennent pour leurs droits. Les années de luttes antérieures à l'ouverture du mariage aux couples homosexuels ont marqué une évolution dans l'affirmation identitaire des familles homoparentales (LGBT). Les deux documentaires produits par l'association témoignent de cette évolution.

51 Dans ses opérations de visibilisation, la FLG a veillé à se présenter en adéquation avec une volonté de normalité. Pour l'association, il s'agissait de montrer des familles 
comme les autres, avec toutes les qualités et tous les problèmes quotidiens relatifs au soin et à l'éducation des enfants. Toutefois, dans cette visée de normalisation des familles homoparentales, il ne se joue pas seulement une réaction à l'hégémonie normative des groupes majoritaires, on y voit également la quête de la reconnaissance d'une spécificité. Cette spécificité de nature identitaire, cherche aussi une valeur ajoutée (Espineira, 2014) ${ }^{18}$, dans la diversité, celle de l'homo et la trans* parentalité. Comme tout à la fois, cette visée normative trace son idéal dans une égalité de droits, ce qui supposait en amont de faire reconnaitre et de rendre identifiables ces familles nouvelles.

\section{BIBLIOGRAPHIE}

BARBOT Janine (1999), « L'engagement dans l'arène médiatique. Les associations de lutte contre le SIDA », Réseaux, n 95, p. 155-196.

BolTANSKI Luc (1990), L'Amour et la justice comme compétences, Paris, Métailié.

CALvo Kerman (2010), « Movimientos sociales y reconocimiento de derechos civiles : la legalización del matrimonio entre personas del mismo sexo en España », Revista de Estudios Politicos, n 147, p. 137-167.

CADORET Anne (2011), « La famille homoparentale en France : une révolution tranquille? », Enfances, Familles, Générations, $n^{\circ} 15$, p. 45-60.

COURDURIÈs Jérôme (2016), « Ce que fabrique la gestation pour autrui », Journal des anthropologues, $\mathrm{n}^{\circ}$ 144-145, p. 53-76.

COURDURIÈs Jérôme (2011), Être en couple (gay). Conjugalité et homosexualité masculine en France, Presses universitaires de Lyon, coll. «SXS Sexualités ».

DÉcHAUX Jean-Hugues (2015), « Intégrer l'émotion à l'analyse sociologique de l'action », Terrains/ Théories [En ligne], n², mis en ligne le 23 octobre 2014, consulté le 27 août 2016.

Descoutures Virginie (2010), Les Mères lesbiennes, Paris, Presses universitaires de France.

Domingo Pauline (2009), « Le quotidien des familles recomposées », Politiques sociales et familiales $\mathrm{n}^{\circ}$ 96, p. 96-104.

DUPUY Claude et Charles HALPERN (2009), « Les politiques publiques face à leurs protestataires ", Revue française de science politique vol. 59, n 4, p. 701-722.

ESPINEIRA Karine (2014), « Les constructions médiatiques des personnes trans - Un exemple d'inscription dans le programme 'penser le genre' en SIC », Les Enjeux de l'information et de la communication $\mathrm{n}^{\circ} 15$, vol. 1, p. 35-47.

FILLIEULE Olivier (2001), « Propositions pour une analyse processuelle de l'engagement individuel », dans FILLIEULE Olivier et Nonna MAYER (sous la dir.), « Devenirs Militants », Revue française de science politique, 51(1)., p. 199-215. 
FILLIEULE Olivier (2014), « Public space, media and protest movements », dans OEHMER Franziska (dir.), Politische Interessenvermittlung und Medien. Funktionen, Formen und Folgen medialer Kommunikation von Parteien, Verbänden und sozialen Bewegungen, Nomos Publisher.

FinE Agnès (1998), Adoptions. Ethnologie des parentés choisies, Paris, Éditions de la Maison des sciences de l'homme.

Gross Martine et Dominique MeHL (2011), « Homopaternités et GPA, Enfances, Familles, Générations, $\mathrm{n}^{\circ} 14$, p. $95-112$.

Gross Martine (2009), « Les familles homoparentales : entre conformités et innovations », Informations sociales, $\mathrm{n}^{\circ} 154, \mathrm{p} .106-114$.

Gross Martine (2012), Choisir la paternité gay, Paris, Ed. ERES.

HÉRAUlT Laurence (2014), La Parenté transgenre, Aix-Marseille, Université de Provence, coll. «Penser le genre ».

HONNETH Axel (2006), « Invisibilité : sur l'épistémologie de la reconnaissance », dans HoNNETH Axel, La Société du mépris, Paris, La Découverte.

KAUfMANN Laurence et Fabienne Malbois (2015), « S'éprouver en public : l'arc affectif de l'indignation dans la controverse 'Iacub-DSK' ", Dans RABATEl A., MonTE M., SOARES RodRIGUes M. (eds.) Comment les médias parlent des émotions : l'affaire Nafissatou Diallo contre Dominique StraussKahn, Lambert-Lucas, p. 99-117.

LEGRAND Stéphane (2007), Les Normes chez Foucault, Paris, PUF, 2007.

MATONTI Frédérique et Franck POUPEAU (2004), « Le capital militant. Essai de définition », Actes de la recherche en sciences sociales, $\mathrm{n}^{\circ} 155$, p. 4-11

MATHIEU Lillian (2004), Comment lutter ? Sociologie et mouvements sociaux, Paris, Textuel, 2004.

Mathieu Lillian (2007), « L'espace des mouvements sociaux », Politix, vol. 1, n 77, p. 131-151.

MATHIEU Lilian (2012), L'Espace des mouvements sociaux, Bellecombes-en-Bauges, Éditions du Croquant.

MEHL Dominique (2011), Les Lois de l'enfantement Procréation et politique en France (1982-2011), Paris, Presses Sciences Po.

MEHL Dominique (2003), « Le témoin, figure emblématique de l'espace public/privé », dans Daniel CEFAÏ et Dominique PASQUIER (dir.), Les Sens du public. Publics politiques, publics médiatiques, Paris, Presses Universitaires de France, 2003 (Coll. « Curapp»), p. 489-502.

NeVeu Erik (2010), « Médias et protestation collective », dans Olivier FillieUle, Éric AGRIKoliANSKY, Isabelle SOMMIER (dir.), Penser les mouvements sociaux. Conflits sociaux et contestation dans les sociétés contemporaines, Paris, La Découverte, p. 245-264.

NEVEu Erik (1999), « Médias, mouvements sociaux et espace public », Réseaux, n . 98, p. 17-85.

PATERNOTTE David (2011), Revendiquer le « mariage gay ». Belgique, France, Espagne, Bruxelles, éditions de l'Université de Bruxelles.

ROCA i ESCODA Marta (2016), « L'expérience juridique de l'action collective. Le cas de l'association catalane Familles Gaies et Lesbiennes ", Nouvelles pratiques sociales, Dossier : « De l'intervention à l'action : Nouvelles avenues d'inclusion des communautés LGBTQI », vol. 28, n 1, p. 87-106. 
RocA i EsCODA Marta (2016), « La procréation partagée des couples lesbiens en catalogne », Journal des anthropologues, $\mathrm{n}^{\circ}$. 144-145, p. 147-168.

Roca i Escoda Marta (2013), « De la dénonciation du droit à la conquête des droits. Le cas des mobilisations homosexuelles ", in FRERE, B et JACQUEMAIN, M., Résister au quotidien ? Presses de Sciences-po, Paris, p. 55-86.

RocA i EscoDA Marta (2011), « De la mobilisation du droit à la réalisation du droit. Les actions de l'association catalane 'Familles Lesbiennes et Gays' », Politix, nº 2, dossier « Discriminations et droit », p. 59-80.

RoCA i EscodA Marta (2010), " Minorités sexuelles et lutte pour la reconnaissance », dans P. CORRIVEAU et V. DAOUST (dir.), La Régulation sociale des minorités sexuelles. L'inquiétude de la différence, Québec, Presses de l'Université de Québec, p. 155-176.

VoIrol Olivier (2005), « Présentation », Réseaux : Communication, technologie, société, vol. 23, $\mathrm{n}^{\circ} 129-130, \mathrm{p}$ 9-36.

\section{NOTES}

1. Dans le cas qui nous occupe, la "dépendance» du mouvement social avec les médias dominants ne se caractérise pas par une déformation médiatique des propos que cette association veut transmettre. Comme nous le montrons dans cet article, la FLG contrôlera la scène d'apparition. Pour une réflexion sur la « dépendance » des mouvements sociaux envers les médias dominants, voir notamment Neveu (2010).

2. Une dizaine d'entretiens a été réalisée, à plusieurs reprises, auprès des personnes clefs, notamment les président.e.s et les membres les plus actifs de l'association.

3. La FLG est la première association de familles homoparentales en Espagne. En 2008, cette association crée l'association de familles LGBT au niveau espagnol. Par la suite, d'autres associations nationales et régionales verront le jour.

4. L'auteure de cet article a traduit librement les récits et documents écrits, du catalan et de l'espagnol au français.

5. En 2010, l'association est composée de 150 membres, qui y adhèrent en participant une première fois à une activité et en payant une cotisation annuelle de 36 euros. La demande d'adhésion se fait formellement par écrit. Dès 2008, l'association reçoit des subventions en tant qu'entité collaboratrice du département d'action sociale et de citoyenneté, du secrétariat de politiques familiales et de la citoyenneté du gouvernement catalan. En 2015, elle se compose de 384 membres.

6. Les huit personnes qui composent le comité ont un niveau d'études particulièrement élevé et exercent des professions intellectuelles supérieures et intermédiaires des secteurs publics, de la santé et du travail social.

7. Leur cause est l'égalité et la protection des enfants et des familles homoparentales. Il y a là un changement important par rapport aux revendications des mouvements homosexuels, dont l'objet de la lutte regardait les droits des personnes homosexuelles elles-mêmes (Armand de Fluvià, 2013 ; Rivero, 2010).

8. La Loi 13/2005 ouvre le mariage aux couples homosexuels. Cependant, les normes de filiation (que cette loi sur le mariage n'avait pas modifiées) ont posé des problèmes de discrimination. Ceux-ci ont été corrigés par deux changements de lois, la Loi de 2006 sur les techniques de reproduction humaine assistée (LTRHA) et la Loi 3/2007 sur l'identité de genre. Avec ces changements législatifs, les couples homosexuels mariés peuvent faire reconnaître une filiation automatique par adoption ou par PMA. 
9. Le mot de pragmatisme est pris ici dans son sens ordinaire, non philosophique.

10. En effet, après plus de vingt ans de domination du parti CIU (de centre-droit et nationaliste), les élections catalanes du 16 novembre 2003 ont vu se former un gouvernement regroupant une coalition tripartite de gauche « catalaniste et progressiste ».

11. Ou codes normatifs (Legrand, $2007: 235$ ), qui montreraient les propriétés de ce qu'est une famille.

12. Le film, réalisé par la journaliste Anna Bolula et produit par la FLG, a gagné divers prix, a été sélectionné dans plus de quarante festivals dans le monde entier, et retransmis sur diverses chaînes de télévision.

13. En 2015, l'association a un compte Twitter, Facebook, Instagram, Vimeo, You Tube, etc. La page Facebook a plus de 3.500 followers; à cela s'ajoute la publication d'un bulletin hebdomadaire.

14. En 2015 l'association comptabilise soixante apparitions dans les médias (radio, TV, journaux nationaux et locaux, presse LGBT, etc.).

15. Nous faisons le choix de ne citer que des auteurs français, mais nous soulignons que cette littérature s'est étendue dans la plupart de contextes nationaux occidentaux.

16. En ce sens, Neveu souligne une intelligence réflexive des enjeux d'une communication maîtrisée par le mouvement social (Neveu, 1999 : 70).

17. Ou « capital militant ", si l'on suit la perspective sur le militantisme de Matonti et Poupeau (2004).

18. L'auteure, en s'intéressant aux effets symboliques de la médiatisation des personnes trans*, cherche à "étudier la façon dont sont diffusées et élaborées les images des transidentités, questionner la réception si possible, définir la part des imaginaires médiatiques et socioculturels, chercher la valeur ajoutée (sociale, morale, esthétique, politique, philosophique) » (Espineira, $2014: 39)$.

\section{RÉSUMÉS}

À partir d'une étude des actions de l'association catalane Familles Lesbiennes et Gaies (FLG) travaillant à la reconnaissance des familles homoparentales, nous focaliserons notre analyse sur les interactions avec les médias, considérés à la fois comme les relais et les médiateurs d'une revendication dans l'espace public. Concernant les formes d'organisation et les activités de la FLG, nous constatons des évolutions et spécificités sur les manières de s'organiser, les objectifs, mais aussi sur la nature et les canaux des revendications. Étant donné le type d'actions envisagées et la nature de la problématisation mise en œuvre (la reconnaissance des familles homoparentales), outre le travail politique auprès des instances juridiques et gouvernementales, la FLG a fait aussi un important travail de visibilisation pédagogique : donner à voir une réalité existante et montrer sa normalité. Sa visée est donc l'acceptation sociale de cette réalité familiale. Pour ce faire, le comité de l'association a logiquement cherché à se doter d'une identité à la fois positive et consensuelle. Ce travail de visibilisation se fait notamment par la voie médiatique, selon deux modalités : la réalisation de documentaires à visée pédagogique et la fabrication d'évènements destinés à être relayés par les médias. Cet article porte sur cette mise en scène à destination des médias et analyse comment la réalité parentale des couples homosexuels est présentée, à l'horizon de sa visibilisation et de sa normalisation. 
By studying the actions of the Catalan NGO Gay and Lesbian Families (LGF), which struggles for the recognition of same-sex parent families, I will analyze their relationships with the media. The media are considered both as a relay and an intermediary to convey the NGO's claims in the public space. Although the organization and activities of LGF have evolved, the NGO is specific on several accounts, regarding its organization, goals, but also the nature and channels of its claims. Given its type of actions and the nature of the problematization that is implemented (the recognition of same-sex families), and besides its political work which targets judicial and state institutions, LGF has done a significant work to make visible, in a pedagogical manner, to show an existing reality and highlight its normality. In so doing, LGF has sought to have this family reality accepted. The NGO's committee has tried to shape a positive and consensual identity. This vibilization work has taken the path of the media using two modes : pedagogical documentaries and the organization of special events. This article deals with the production of this visibility in the media and it analyzes how the reality of same-sex parents is therefore set out in order to make it visible and normal.

\section{INDEX}

Mots-clés : familles homoparentales, visibilisation, normalisation

Keywords : same-sex parents, visibilisation work, making normal 